\title{
El daño cerebral sobrevenido: un abordaje transdisciplinar dentro de los servicios sociales
}

\section{Acquired brain damage: a transdisciplinary approach within social services}

INMACULADA GÓMEZ PASTOR*

\begin{abstract}
RESUMEN
La población con Daño Cerebral Adquirido va siendo cada vez más numerosa, siendo los jóvenes ( entre 15 y 24 años ) más susceptibles de sufrir lesiones cerebrales. Es imprescindible llevar a cabo una rehabilitación especializada en el Daño Cerebral, con equipos de profesionales que compartan conocimientos y objetivos. La rehabilitación transdisciplinar facilitará a la persona afectada la consecución del nivel más alto de adaptación fisica, psicológica y social posible, incluyendo todas las medidas necesarias para que la persona afectada pueda conseguir una integración óptima, como indica la OMS. Esto se puede conseguir desde varios ámbitos de actuación uno de los cuales es el de los Servicios Sociales.
\end{abstract}

\section{PALABRAS CLAVE}

Daño cerebral sobrevenido, Accidente Cerebro Vascular (ACV), Traumatismo Craneoencefálico (TCE), Rehabilitación.

\section{ABSTRACT}

There is an increased incidence of acquired brain damage, with an especial impact in young individuals with ages ranging between 15 and 24 ages old. In order to achieve the best possible integration of affected persons, as pointed out by the WHO, it is necessary to

* Doctora en Psicología. Máster en Neuropsicologia. Directora Gerente del CEADAC (IMSERSO). 
implement specialized rehabilitation programs, that include professionals from different areas sharing their knowledge and objectives. Such transdisciplinar rehabilitation process will facilitate the physical, psychological and social adaptation to the highest possible level. The Social Services are one of the ways to reach this objective.

\section{KEY WORDS}

Acquired Brain Damage (ABD), Cerebrovascular Accident (CVA), Traumatic Brain Injury (TBI), Rehabilitation. 


\section{INTRODUCCIÓN}

El daño cerebral sobrevenido es el resultado de una lesión súbita, en la mayoría de los casos, en las estructuras cerebrales, que da lugar a diferentes problemas de muy diversa índole, ya que el cerebro controla todas las funciones vitales desde las más básicas (respiración, presión arterial, frecuencia cardiaca), hasta las más sofisticadas (creación artística, abstracción, emociones), no obstante no disponemos de un mapa funcional consensuado y cerrado (Modelo de atención a las personas con daño cerebral, IMSERSO 2007).

El estudio epidemiológico del daño cerebral únicamente cuenta con estudios de incidencia de Accidentes Cerebro Vasculares (ACV), y Traumatismos Craneoencefálicos (TCE), especialmente en poblaciones anglosajonas, sin embargo estos estudios carecen de información sobre la prevalencia (número de casos con un determinado problema en un punto en el tiempo) de las discapacidades resultantes del daño cerebral.

De la Encuesta de discapacidades y deficiencias y estado de la salud realizada en el año 1999 podemos extrapolar los siguientes datos de prevalencia: Aproximadamente 570 personas por 100.000 habitantes y año presentan una discapacidad secundaria al daño cerebral adquirido (DCA). El 83\% son ACV y el $19,5 \%$ son debidos a TCE.

El Defensor del pueblo en su informe publicado con el título "Daño cerebral sobrevenido en España: un acercamiento epidemiológico y socio sanitario", refiere una incidencia anual de ictus de 266 por cada 100.000 habitantes de los que al menos una tasa de 143 por 100.000 habitantes tendrán posibilidades de presentar secuelas moderadas o graves. El
$23 \%$ de estos enfermos tienen una edad inferior a 65 años.

Así mismo se estima una tasa de incidencia de 100.000 nuevos casos de TCE por 100.000 habitantes y año de los que 15 de cada 100.000 habitantes/año tendrán secuelas moderadas y graves.

Aproximadamente 300.000 personas sufren en España una discapacidad cuyo origen es el DCA. Estos datos empujan al estudio de esta población desde el punto de vista tanto de la rehabilitación como de la atención para poder mejorar su calidad de vida.

\section{LA REHABILITACIÓN DEL DAÑO CEREBRAL ADQUIRIDO DENTRO DE LOS SERVICIOS SOCIALES}

Una reflexión que debemos hacer es si la rehabilitación de las personas con Daño Cerebral Adquirido debe establecerse dentro del área de los servicios sociales o debería estar en un contexto sanitario.

Entendiendo que la misión de los Servicios Sociales consiste en la provisión de servicios y prestaciones a la ciudadanía, o a determinados sectores específicos de la misma para lograr su bienestar social o incrementarlo y que desarrollan esta misión mediante servicios que comprenden la prevención de las situaciones de exclusión o desventaja social, la asistencia propiamente dicha y la rehabilitación y reinserción social de los problemas atendidos, cabe entender que la rehabilitación de las personas con Daño Cerebral Sobrevenido puede estar perfectamente encuadrada en el ámbito de los Servicios Sociales. Como se ha dicho anteriormente uno de los servicios que prestan es la rehabilitación y reinserción social de personas en situación de exclusión o desventaja social. Podemos afir- 
mar que la población sujeto de este artículo está encuadrada en estos parámetros.

Los principales servicios del Sistema de Servicios Sociales se engloban en diferentes áreas, una de las cuales son los centros de especialidades y referencia, existiendo los servicios sociales especializados que son aquellos cuyas prestaciones implican intervenciones de carácter sectorial, atendiendo a la tipologia de las necesidades, que requieren una especialización técnica o la disposición de recursos determinados. Además no podemos olvidar la Ley de Promoción de Autonomía Personal y Atención a las Personas en Situación de Dependencia, (Ley 39/2006 del 14 de diciembre), en cuyo nombre aparece la base de la rehabilitación, que no es otra cosa que la promoción de la autonomía personal.

No voy a negar el carácter sanitario de la rehabilitación del Daño Cerebral Adquirido, pero tampoco puedo privar de los beneficios que puede darle el aspecto social, en tanto en cuanto esta patología produce además del daño corporal, discapacidades e incluso dificultades de inserción en la comunidad, objeto de actuación claro de los Servicios Sociales.

En el año 2002 se inaugura el Centro de Referencia Estatal de Atención al Daño Cerebral (CEADAC), creado por el IMSERSO (Orden TAS/55/2002 de 8 de enero, BOE de 17 de enero de 2002), cuya misión es promover en todos los territorios del Estado recursos especializados para la rehabilitación integral e intensiva de personas con lesión cerebral adquirida y no progresiva. Para cumplir esta misión el CEADAC cuenta con un área de referencia que pone a disposición de las instituciones y profesionales que trabajan en la atención del DCA, información, documentación, asesoría y consultoría. Cuenta también con un área de atención directa que ofrece servicios de asistencia personalizada, con rehabilitación intensiva para la autonomía personal, que comprende rehabilitación médico funcional, fisioterapia, terapia ocupacional, logopedia, neuropsicología y trabajo social. Además existe un servicio de apoyo y formación a familiares.

El CEADAC forma parte de la Red de Centros de Referencia Estatales del IMSERSO (CRE), que a su vez se integra en el Sistema para la Autonomía y Atención a la Dependencia (SAAD), que es un subsistema del Sistema de Servicios Sociales. Mediante la creación del SAAD, la Ley de Promoción de la Autonomía Personal y Atención a las Personas en Situación de Dependencia regula las condiciones básicas que garanticen la igualdad en el ejercicio del derecho subjetivo de ciudadanía a la promoción de la autonomía personal y atención a las personas en situación de dependencia.

Los CRE están concebidos como centros de alta especialización en materia de prevención, promoción de la autonomía personal y atención integral a colectivos de personas mayores y/o de personas con discapacidad en situación o en riesgo de dependencia. Son centros avanzados en la promoción y desarrollo de conocimientos, gestión de calidad, experiencias y métodos de intervención, especializados en una materia o colectivo determinado, que persiguen apoyar la excelencia de los Servicios Sociales.

De acuerdo con su misión el CEADAC tiene los siguientes objetivos de actuación:

- La rehabilitación sociosanitaria de las personas con Daño Cerebral Sobrevenido

- El impulso en todos los territorios del Estado de la rehabilitación y la 
mejora de la calidad de vida de dichos afectados y sus familias.

- La información y asistencia técnica a las Administraciones Públicas, Instituciones, Entidades públicas o privadas y a cuantas personas 10 soliciten sobre la atención y rehabilitación socio sanitaria de personas con discapacidad con Daño Cerebral Adquirido.

Después de esta descripción, queda probado el lugar que puede tener dentro de los Servicios Sociales la rehabilitación de personas con Daño Cerebral Adquirido, en tanto en cuanto estamos hablando de una población con la que se debe trabajar la promoción de la autonomía personal, como indica el título de la ley 39/2006 del 14 de diciembre, sin dejar de prestar atención a la segunda parte de este mismo título, la atención a las personas en situación de dependencia, situación en la que puede estar la población de referencia.

\section{EL EQUIPO DE REHABILITACIÓN}

Por todos es aceptada la necesidad de abordar la rehabilitación del daño cerebral, desde diferentes disciplinas, ya que suscita problemas de diferente índole, sin embargo quisiera reflexionar sobre lo que esto implica, no sólo en cuanto a componentes del equipo sino en cuanto a forma de funcionar.

Existen múltiples definiciones de trabajo en equipo. Elegiré la que hace referencia a la actividad de un grupo de personas que realizan distintas aportaciones hacia el logro de un objetivo común; es importante resaltar la necesidad de interacción entre los diferentes componentes del grupo, y no resignarnos con sumar las diferentes opiniones, esto me lleva a entrar en la clasificación en equipos multidisciplinares, equipos interdisciplinares y equipos transdiciplinares.

El equipo multidisciplinar hace referencia a la interacción entre varias disciplinas, esto se concreta desde la mera comunicación hasta una verdadera integración, en cuanto a metodología, objetivos, terminología etc. Se persigue una intervención conjunta por parte de todos los miembros del equipo, que prima sobre las intervenciones individuales.

El equipo interdisciplinar hace referencia a un conjunto de profesionales, igualados entre si que quieren conseguir un objetivo común, aportando cada uno sus conocimientos, experiencias etc., de tal manera que mediante intercambios e integraciones mutuas se genere un enriquecimiento de todos los componentes. Es fundamental fijar objetivos comunes y específicos que pretenderán resolver el problema cuya solución sólo se encontrará en el seno del equipo.

El equipo transdisciplinar corresponde a la sucesión de relaciones interdisciplinarias integradas de modo que el sistema resultante no tendría fronteras sólidas entre las disciplinas. Se basa en que "la naturaleza es única y se la intenta conocer prescindiendo de las divisiones introducidas en la Ciencia por el hombre".

Si bien es cierto que la persona es única, su abordaje también debe ser único y por lo tanto transdisciplinar, sin embargo me quedaré con la necesidad del abordaje multidisciplinar o interdisciplinar, de la rehabilitación del Daño Cerebral Sobrevenido, en tanto en cuanto se trabajarán desde diferentes disciplinas hacia la consecución de un objetivo común, sin embargo no podemos olvidar que si bien es cierto que" los equipos multidisciplinares que abordan tareas 
complejas, son de un potencial y una riqueza humana extraordinarios, su correcto funcionamiento requiere aptitudes caracteriales en los miembros del equipo que los orienten a la identificación de un objetivo común, a la cohesión del grupo y a la superación de inercias previas inherentes a la disciplina de la que proceden" ( Quemada J.I., 2007).

El Daño Cerebral Adquirido, es una compleja realidad en la que aparecen una gran cantidad de áreas a rehabilitar, tanto en el aspecto motor, como psicológico (incluyendo en éste el ámbito cognitivo, emocional y familiar), sin olvidar el aspecto social.

La definición de la OMS de rehabilitación implica que el paciente pueda llegar a conseguir el nivel más alto de adaptación física, psicológica y social posible, incluyendo todas las medidas necesarias para que la persona afectada pueda conseguir una integración óptima. Esta definición se muestra en todo su esplendor en los casos de DCA, en los que verdaderamente el déficit es global y que obliga a centrar el tratamiento en la persona, en tanto en cuanto no hay dos casos iguales. Así mismo, no hay que olvidar las necesidades de la familia, que no deja de estar afectada igualmente y en ocasiones incluso de forma más dramática que la propia persona, siendo preciso ofrecer un apoyo tanto informativo como terapéutico.

Teniendo en cuenta la diversidad de áreas que se pueden ver involucradas en el daño cerebral, es preciso desarrollar programas de rehabilitación individualizados que recojan la peculiaridad de cada caso y se ajusten a sus necesidades desde todas las perspectivas posibles.

Siguiendo el Modelo de atención a las personas con Daño Cerebral (IMSERSO 2007), las áreas que se trabajan en la rehabilitación del Daño Cerebral Adquirido son:

- Nivel de alerta.

- Control motor.

- Recepción de información.

- Comunicación.

- Cognición.

- Emociones y personalidad.

- Actividades de la vida diaria.

Esto hace que el DCA deba ser abordado por un equipo multidisciplinar, debido a la complejidad y multidimensionalidad de las secuelas, siendo necesaria la participación de numerosas disciplinas en un momento $\mathrm{u}$ otro del proceso rehabilitador: médico rehabilitador, neurólogo, neurocirujano, internista, urólogo, traumatólogo, fisioterapeuta, terapeuta ocupacional, psicólogo, neuropsicólogo, logopeda, dietista, farmacólogo, profesional de la enfermería, trabajador social..... (Bernabeu M., Roig T. 1999).

Cada profesional se deberá responsabilizar del área de su competencia de tal manera que el resultado no sea una mera suma de metas de tratamiento sino una respuesta integrada, dando lugar a un programa en el que se hayan priorizado intervenciones y acordado objetivos. Este programa será implementado, en la medida de lo posible, por todo el equipo y tendrá en cuenta la opinión y participación de la persona con daño cerebral y su familia (Bori, I., Gangoiti L., Marín J. Guemada J.I., Ruiz M. J, 2007).

\section{El equipo médico}

Es el responsable del manejo clínico y debe coordinar las acciones del resto del equipo realizando las exploraciones clínica, neurológica y funcional, valorando los déficits y discapacidades, además debe establecer los objetivos y orientar los diferentes programas terapéuticos de 
las diferentes disciplinas, realizando el seguimiento y la consecución de objetivos. Así mismo debe realizar una labor de prevención, con una doble labor, por una parte educacional hacia la familia que debe conocer las medidas preventivas adecuadas y por otra parte indicando el tratamiento y las pruebas complementarias que pueden ser necesarias para el manejo de determinadas complicaciones cerebrales.

También aplicará técnicas específicas de su competencia.

El papel del especialista en psiquiatría, si existen problemas conductuales, y del neurólogo o neurocirujano, también son importantes.

\section{El Equipo de Enfermeria}

Además de sus funciones habituales, cumplen un papel de asesores de la persona con DCA y sus familias, adiestrándoles en diferentes técnicas para la reinserción domiciliaria. Trabajan en colaboración con el resto del equipo para restaurar y mantener la salud física, y social.

\section{Fisioterapia}

$\mathrm{Su}$ principal objetivo es conseguir el mayor grado de autonomía física, optimizando los recursos motores, con diferentes técnicas de movilización, de adquisición y mejora del equilibrio de reeducación de las alteraciones de la coordinación, tratamiento de las alteraciones del tono muscular etc. Debe estar familiarizado con los productos de apoyo relacionados con la marcha.

\section{Terapia Ocupacional}

Interviene en la consecución del mayor grado de autonomía en las actividades de la vida diaria, mediante programas de estimulación multisensorial, entrenamiento en la dominancia de la mano si fuera necesario, adaptación y modificación del entorno doméstico, tratamiento de la coordinación visuo manual, valoración, asesoramiento, y selección de adaptaciones personales y productos de apoyo etc.

\section{Logopedia}

$\mathrm{Su}$ responsabilidad se centra en el tratamiento de los trastornos del habla y lenguaje, sin olvidar el abordaje que realizan de los problemas de deglución. Las principales áreas de trabajo son la evaluación y rehabilitación de la disfagia, la disfonia, la disartria, las afasias, los trastornos pragmáticos de la comunicación, la colaboración en los programas de estimulación multisensorial y la implantación y entrenamiento en el uso de sistemas alternativos y aumentativos de la comunicación.

\section{Trabajo social}

$\mathrm{Su}$ área de actuación se centra en el entorno socioeconómico del paciente, con el fin de realizar una reinserción familiar y social lo mejor posible, informando sobre los trámites a realizar para solicitar ayudas prestaciones, reconocimiento de minusvalia, incapacidad laboral etc., además da información sobre los recursos sociales disponibles.

\section{Neuropsicologia}

Tradicionalmente, se entendia por neuropsicologia las relaciones entre conducta y cerebro.

A finales de los años sesenta se pro- 
duce un encuentro entre la neuropsicología y la ciencia cognitiva, que concibe el cerebro como un sistema de procesamiento de la información generando modelos de dicho procesamiento para cada función cognitiva, de tal manera que la neuropsicología es la disciplina que explica las alteraciones conductuales de un paciente neuropsicológico en la vida cotidiana, tras la lesión cerebral, en términos de su patrón de funcionamiento cognitivo, y como este patrón interactúa con las variables psicológicas no cognitivas de ese paciente.

Los profesionales de la neuropsicología establecerán un programa de rehabilitación de las funciones cognitivas (orientación, atención y control ejecutivo, comunicación y lenguaje, funciones visuoespaciales, visoperceptivas y gnósicas, funciones visoconstructivas, gnosias digitales, praxias gestuales, cálculo, funciones del pensamiento, y funciones de aprendizaje y memoria) siempre con base en una evaluación previa y entendiendo que el objetivo de la rehabilitación neuropsicológica es tratar al paciente en tanto que individuo en su entorno cotidiano (Benedet, 2002).

De esta manera el abordaje rehabilitador del Daño Cerebral Adquirido adquiere una dimensión transdisciplinar, que se nutre de diferentes disciplinas y cuyo objetivo es la promoción de la autonomía personal, intentando una total reinserción en la comunidad.

\section{BIBLIOGRAFÍA}

Benedet M.J. (2002). Fundamento Teórico y Metodológico de la Neuropsicología Cognitiva, Madrid, IMSERSO.

Bernabeu M., Roig T. (1999). La rehabilitación del traumatismo craneoencefálico: un enfoque rehabilitador Barcelona. Fundació Institut Guttmann,

Bori, I., Gangoiti L., Marín J. Quemada J.I., Ruiz M. J. (2007). Modelo de atención a las personas con Daño Cerebral, Madrid, IMSERSO.
Daño cerebral sobrevenido en España, un acercamiento epidemiológico y sociosanitario. Informe del Defensor del pueblo (2005). Madrid

Guía de familias (2006). Federación Española de Daño Cerebral Adquirido (FEDACE).

Quemada J.I., (2007). Papel del psiquiatra en un servicio de Daño Cerebral. Revista Colombiana de psiquiatría, Vol. 36 , suplemento no 1 . 